\title{
Sliding Mode Control of Grid Chaotic Oscillation based on Immune Algorithm
}

\author{
Li Xiangqun ${ }^{1, *}$, Liu Jianhua ${ }^{2}$ and Yang Longyue ${ }^{2}$ \\ ${ }^{1}$ School of Computer Science and Technology, China University of Mining and Technology, Xuzhou 221008, China \\ ${ }^{2}$ School of Information and Electrical Engineering, China University of Mining and Technology, Xuzhou 221008, China
}

Received: 24 Aug. 2013, Revised: 26 Nov. 2013, Accepted: 27 Nov. 2013

Published online: 1 Jul. 2014

\begin{abstract}
The bifurcation and chaos phenomenon which exist in power grid are threats to the stable operation of power grid and the safety of people and equipment. In order to eliminate the chaos phenomena in power grid, a kind of non-singular terminal sliding mode (NTSM) variable structure control method based on immune algorithm is put forward. The second-order model of grid chaotic oscillation is built and to be controlled by non-singular terminal sliding mode variable structure controller. Meanwhile, the immune algorithm is introduced to optimize the sliding mode controller parameters. By means of simulation, the effectiveness of this method in the grid chaos control is verified.
\end{abstract}

Keywords: Power Grid, Chaos, Non-singular Terminal Sliding Mode, Immune Algorithm.

\section{Introduction}

Modern power system is a complex system of strong nonlinear coupling and inevitable uncertainty. In addition to periodic oscillations of the normal operation, there are complex bifurcation and chaos running behaviors [1,2, 3], showing as ferromagnetic resonance, chaotic oscillations, sub-synchronous resonance or voltage collapse phenomenon. This chaos has adverse effects on the safe operation of the grid [4]. Over-voltage and over-current occur and sometimes stably exist, which will cause insulation flash-over, lightning pipe explosion, equipment damage and power outages. The serious damage to valuable equipment like shaft can be caused by grid running state in the unstable limit cycles or chaotic attractors in just a few minutes. The stability of the grid operation and the safety of people and equipments are under serious threats.

At present, a lot of the chaotic phenomena control methods have been proposed by scholars both at home and abroad, such as delayed feedback [5], adaptive control [6], and intelligent control [7] and so on, some of which have been applied in the field of engineering practice. Many advanced control theories, methods and techniques have been introduced to improve grid security and stability. Most of these methods guide the power system to the normal operation $[8,9,10]$ from the chaotic state through injecting the energy of active power and reactive power into the system. The proposal and application of these methods provide a reliable guarantee for the safe operation of the system.

As a strong nonlinear system, the grid chaotic system can be controlled to a steady state by conventional control methods with poor dynamic control performance. The existence of the severe transition process buffeting, long transition process time and large control energy seriously affect the dynamic control performance. Therefore, it is both a key issue in the power system of chaos control to develop grid stability and an important way to improve the dynamic control effect of the grid chaotic system.

According to the strong nonlinear characteristic of the grid chaotic model, an adaptive immune genetic algorithm based non-singular terminal sliding mode variable structure control method of the grid is put forward. The secondorder model of grid chaotic oscillation is built to adapt to how the non-singular terminal sliding mode variable structure controller is designed. On this basis, the adaptive immune genetic algorithm is used to optimize the sliding mode controller parameters for the chaotic system. By the simulation of applying this optimized sliding mode controller to the chaotic model of the grid, the validity and accuracy of the method can be checked.

\footnotetext{
* Corresponding author e-mail: cumtliujh@gmail.com
} 


\section{The nonlinear dynamics analysis of the grid}

In order to analyse the principle of the chaotic oscillation, external factors such as disturbance of the system are mainly considered. But the effects of the internal factors including differences of each end-side equivalent moment are not taken into consideration. The high voltage power grid [11] can be described as Figure 1.

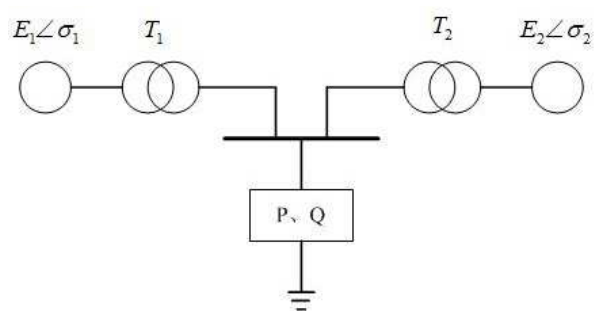

Figure 1: Power grid equivalent system

According to Figure 1, the grid second-order model under the electromagnetic power disturbances can be acquired:

$$
\left\{\begin{aligned}
& \frac{d \sigma(t)}{d t}=\omega(t) \\
& H \frac{d \omega(t)}{d t}=-P_{\max } \sin \sigma(t)-D \omega(t)+P_{m} \\
&+P_{\zeta} \cos \beta t-\delta \cos k t \sin \sigma(t)
\end{aligned}\right.
$$

$\sigma(t)=\sigma 1-\sigma 2$ is the relative angle between the q-axis electric potential of the system 1 and 2 equivalent generators ( $\mathrm{rad}) ; \omega(t)$ is the relative angular velocity $(\mathrm{rad} / \mathrm{s}) ; \mathrm{H}$ is the equivalent moment of inertia $(\mathrm{kg}$ ? $\mathrm{m} 2)$; D is the equivalent damping coefficient $(\mathrm{N}$ ?m? $/ \mathrm{rad}) ; P_{\max }$ is the maximum electromagnetic power transmitted from system 1 to system $2(\mathrm{~W}) ; P_{m}$ is the equivalent generator 1 mechanical power (W); $P_{\xi}$ is the disturbance power amplitude (W); $\beta$ is the disturbance power frequency $(\mathrm{Hz}) . \delta=\Delta p P_{\max }$ is the electromagnetic power disturbance amplitude; $\Delta p$ is increasing multiples of the electromagnetic power. There are two disturbances in the system, the amplitude of which are $P_{\xi}$ and $\delta$.

In order to demonstrate the system state, the parameters are substituted as: $k=0.8 \mathrm{~Hz}, P_{\xi}=2 \mathrm{~W}, P_{\max }=$ $100 \mathrm{~W}, \mathrm{H}=100 \mathrm{~kg} \cdot \mathrm{m} 2, P_{m}=20 \mathrm{~W}, \mathrm{D}=40 \mathrm{~N} \cdot \mathrm{m} \cdot \mathrm{s} / \mathrm{rad}, \beta=$ $1 \mathrm{~Hz}$, initial value of $\sigma, \omega: p i / 15,0$. The grid operation state under the electromagnetic power disturbance can be acquired. When $\sigma=0$, the system works properly. When $0<\sigma<127$, with the disturbance amplitude increasing, the state of the period-doubling bifurcation appears. When $\sigma \geqslant 127$, the system is in chaos state. The $\sigma, \omega$ timing diagrams when $\sigma=127$ are shown in Figure 2 .

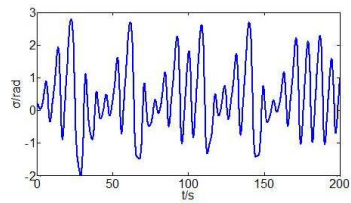

(a) timing diagram of the power angle $\sigma$

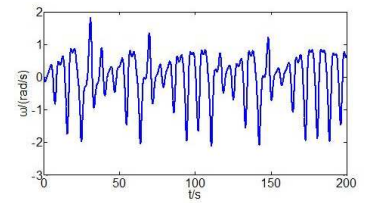

(b) timing diagram of the angular velocity $\omega$
Figure 2: Chaotic running state $(\delta=127)$

\section{The design of grid chaos sliding mode controller}

According to the system operation, sliding mode control with nonlinear nature switches to drive the system to the sliding mode. A large number of researches show that the sliding mode variable structure control works well in nonlinear control. Accompanied by other control methods, the sliding mode variable structure control can solve many problems. Therefore, the sliding mode variable structure control is introduced to the grid chaotic behavior control for the suppression and elimination of the bifurcation and chaotic phenomenon.

\subsection{The design of non-singular terminal sliding mode variable structure controller}

When traditional linear sliding mode control reach the sliding mode state, the system state approaches the origin exponentially along the designed exponentially progressive, which cannot converge the steady-state error to zero in the limited time. Therefore, instead of the traditional linear sliding mode nonlinear sliding mode, the improved non-singular terminal sliding mode is chosen to converge the system state to the equilibrium point in finite time. For the general second-order system:

$$
\left\{\begin{array}{l}
\dot{x_{1}}=x_{2} \\
\dot{x_{2}}=f(x, t)+u(t)+d(t),
\end{array}\right.
$$

Where $x(t)=[x 1(t), x 2(t)]^{T} \cdot f(x, t)$ is disturbance in the system with unknown function. Here its estimated value is assumed $\hat{f}(x, t)$, and suits $|f(x, t)-\hat{f}(x, t)| \geqslant$ $F(x, t) . \mathrm{d}(\mathrm{t})$ is the system external disturbance, which is assumed that $|d(t)| \geqslant D$.

The design of non-singular terminal sliding mode is as follow.

$$
S(t)=x_{1}+\frac{1}{\beta} x_{2}^{p / q}
$$

$\beta>0, \mathrm{p}$ and $\mathrm{q}$ are odd, and $1<p / q<2$. Based on sliding mode equivalent control theory, control law is design as follows.

$$
u=u_{e q}+u_{n}
$$


$u_{e q}$ is equivalent control, and $u_{n}$ is nonlinear control.

Taken no account of the system parameter perturbation and external disturbance, the first derivative of the sliding mode $\mathrm{S}$ is searched.

$$
\begin{aligned}
\dot{S}(t) & =x_{2}+\frac{p}{\beta q} x_{2}^{(p / q-1)} \dot{x}_{2} \\
& =x_{2}+\frac{p}{\beta q} x_{2}^{(p / q-1)}\left(\hat{f}(x, t)+u_{e q}(t)\right) \\
& =\frac{p}{\beta q} x_{2}^{(p / q-1)}\left[\hat{f}(x, t)+u_{e q}(t)+\frac{\beta q}{p} x_{2}^{(2-p / q)}\right] \\
& =0
\end{aligned}
$$

The equivalent control can be got as

$$
u_{e q}(t)=-\hat{f}(x, t)-\frac{\beta q}{q} x_{2}^{(2-p / q)}
$$

For the grid second-order chaotic system, in order to converging $(\sigma, \omega)$ to the point $(0,0)$ quickly, the nonsingular terminal sliding mode control is used.

$$
S(t)=\sigma+C \omega^{p / q}
$$

The equivalent control can be calculated according to grid second-order chaotic system model.

$$
\begin{aligned}
\dot{S}(t) & =\omega+C \frac{p}{q} \omega^{\left(\frac{p}{q}-1\right)} \dot{\omega} \\
& =\omega+C \frac{p}{q} \omega^{\left(\frac{p}{q}-1\right)}\left[\frac { 1 } { H } \left(-P_{\max } \sin \sigma-D \omega+P_{m}\right.\right. \\
& \left.\left.+P_{\zeta} \cos \beta t-\delta \operatorname{coskt} \sin \sigma\right)+u_{e q}\right] \\
& =C \frac{p}{q} \omega^{\left(\frac{p}{q}-1\right)}\left[\frac { 1 } { H } \left(-P_{\max } \sin \sigma-D \omega+P_{m}\right.\right. \\
& \left.\left.+P_{\zeta} \cos \beta t-\delta \cos k t \sin \sigma\right)+u_{e q}+\frac{q}{C p} \omega^{\left(2-\frac{p}{q}\right)}\right] \\
& =0
\end{aligned}
$$

The equivalent control can be got as follow.

$$
\begin{aligned}
u_{e q}(t) & =-\frac{1}{H}\left(-P_{\max } \sin \sigma-D \omega+P_{m}+P_{\zeta} \cos \beta t\right. \\
& -\delta \cos k t \sin \sigma)+u_{e q}-\frac{q}{C p} \omega^{\left(2-\frac{p}{q}\right)}
\end{aligned}
$$

$\mathrm{C}$ is the biggest dynamic effect factor for the reaching segment. When $\mathrm{C}$ is small, the initial requirement of the energy cost is too high. When $\mathrm{C}$ is large, control output is smaller, but it costs a long time to control the system to stable state from the chaotic state. Therefore it should be selected in consideration. law.

Nonlinear control is selected as power rate reaching

$$
u_{n}(t)=-\varepsilon \operatorname{sgn}(S)-k|S|^{\frac{q}{p}} \operatorname{sgn}(S)
$$

$$
\varepsilon>0, k>0 .
$$

In the power rate reaching law, parameters and $\mathrm{k}$ are selected appropriately to ensure the fast reaching speed and suppression of high frequency chattering. A small value of can effectively suppress the high frequency chattering, which will spend a long time for the system state to get into the sliding mode when $|S|<1$. Too small will weaken the robustness of sliding mode control. An amplified $\mathrm{k}$ can speed approaching process with defects that increase the required control strength and engender the high frequency chattering. But a small value of $\mathrm{k}$ will decrease the speed that approaches to the sliding mode. It is clear that the reasonable selecting of power rate reaching law parameters is an important factor affecting system performance.

\subsection{Parameter optimization based on adaptive immune genetic algorithm}

The controller can achieve good control result only with the appropriate parameters. There are some experienced tuning formulas for linear systems, but no available tuning formulas for the non-linear systems. Starting from the control theoretical point of view, the non-linear controller parameters can be optimized with the help of genetic algorithms $[12,13,14]$ according to the physical meaning of the optimal index.

Immune function of the biological immune system can be described as invasive antigen adaptive recognition and elimination. By introducing the learning, memory, and recognition characteristics of biological immune system to genetic algorithm, general individuals are vaccinated with vaccines extracted from the best individual gene. In this way, the global optimal solution can be quickly found just using effective local features in some complex conditions.

Immunization operation is divided into immune vaccinating and immune selection. The former can improve the fitness, and the latter can prevent the degradation of population. Immune operation vaccinates the low fitness individuals in population with excellent selected vaccine to enhance the overall performance. The immune operations are implemented in a certain part or several parts.

On the initial stage, the number of injection points is increased to accelerate the convergence speed and obsolete poor individuals as soon as possible. In the meantime, the number of individuals to be vaccinated should be appropriately reduced in order to maintain the diversity of the population. In a later stage, the injection points should be properly reduced to ensure the population convergence. And in order to avoid the algorithm stagnated, the number of vaccinated individuals should be increased. Based on this principle, adaptive numbers of vaccinated individuals and vaccination points are designed as follow.

The number of vaccinated individuals is

$$
S N(t)=\alpha N /\left(1+e^{-\beta t}\right), 0<\alpha<1,0<\beta<1
$$


The number of vaccination points is

$$
p(t)=e^{-t^{2} / 2 \sigma^{2}}, \sigma=T / 3
$$

$\mathrm{N}$ is the sample number. And $\mathrm{T}$ is the termination time of evolution.

Non-singular terminal sliding mode variable structure controller involves many parameters and logical judgments. With too many parameters taken into optimization, the genetic algorithm convergence rate will be slow, and the optimal parameters will not be searched quickly. Sometimes the genetic algorithm may fall into local extreme point, which has a serious impact on the control effect. To ensure effectiveness and simplicity, the $\mathrm{C}$ in equivalent control, and $\mathrm{k}$ in non-linear control are chosen as three parameters for optimization.

For controller parameters optimization, the required parameters for GA solving are as follows.

(1) Determine the solution space. In order to prevent excessive control energy, the scopes of parameters are limited as follows. The range of parameters $\mathrm{C}$, and $\mathrm{k}$ are selected respectively as $[0,1],[0,0.01]$ and $[0,5]$. (2) Determine the fitness function and the objective function. In order to obtain satisfactory control performance, the minimum absolute value of the sliding mode function is used as the performance index. Meanwhile the square of the input is added to the fitness function to prevent excessive control energy.

The fitness function is defined as follow.

$$
J=\min \int_{0}^{\infty} e^{2}(t) \mathrm{d} t
$$

Parameter e is integral deviation of the angle $\sigma$.

The objective function is taken as the reciprocal of the fitness function.

$$
J=\max \left(\frac{1}{\int_{0}^{\infty} e^{2}(t) \mathrm{d} t}\right)
$$

(3) Determine the selection, crossover, and mutation operator. The proportion selection operator is chosen as selection operator. Single-point crossover operator is selected as crossover operator, and the basic bit mutation operator is used as mutation operator.

(4) Determine the operating parameters of the genetic algorithm, with 100 taken as population size, 30 as number of evolution generation, 0.6 as crossover probability, 0.1 as mutation probability.

After multiple generations of evolution, the optimal controller parameters $[\mathrm{C}, \varepsilon, \mathrm{k}]=[0.5212,0.0096,4.9543]$ are achieved.

\section{Grid chaos control simulation}

These parameters are used to control the second-order model of the power system. First of all, the grid works in the chaotic state without control. In order to suppress this chaotic phenomenon and calm the chaotic state to the steady state, controller is put into system in fifth second. Matlab software ode45 simulation is used with step size $0.001 \mathrm{~s}$. The 100s simulation result is shown in Figure 3.

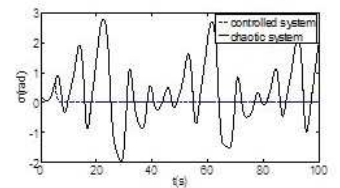

(a) timing diagram of the power angle $\sigma$

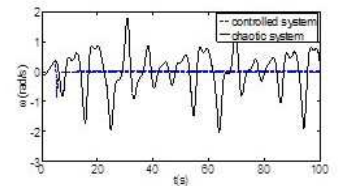

(b) timing diagram of the angular velocity $\omega$

Figure 3: The simulation results

It can be seen from the control curve and the sliding surface $S$ that the system is chaotic before fifth second. After the input of controller, the quantity of system state reaches a small neighborhood near the sliding surface without oscillation. The steady state is achieved after 2 seconds of transition process. And then chaos is eliminated soon. The system reaches the preset steady state in a short time.

\section{Conclusions}

In order to solve the regular grid chaos problems of long control time, poor dynamic effects and large control energy, a non-singular terminal sliding mode variable structure control method based on adaptive immune genetic algorithm is proposed. The second-order model of grid chaotic oscillation is established to adapt to the designed non-singular terminal sliding mode variable structure controller. The equivalent control is designed and non-linear control is selected as the power reaching law. In order to further improve the control dynamic effect, the adaptive immune genetic algorithm is introduced to optimize the parameters of the sliding mode controller. The minimum absolute value of sliding mode function is used as the performance index. In addition, in order to prevent the excessive control energy, the square of the control input is added to fitness function which ensures the best dynamic control effect. The simulation is carried out to control the grid with this optimized sliding mode controller. The results show the methods validity and accuracy, which provides a good solution to the problem of poor dynamic control effects.

\section{Acknowledgements}

This work is supported by the 973 Program (No. 2013CB329502), the National Natural Science Foundation (No. 61379101), the Basic Research Program (Natural Science Foundation) of Jiangsu Province of China 
(No. BK20130209), the Opening Foundation of the Key Laboratory of Intelligent Information Processing of Chinese Academy of Sciences (IIP2010-1), and the Opening Foundation of Beijing Key Lab of Intelligent Telecommunications Software and Multimedia, Beijing University of Posts and Telecommunications.

\section{References}

[1] Iravani, M. R., Chaudhary, A. K. S., Giesbrecht, W. J., Hassan, I. E., Keri, A. J. F., Lee, K. C., Martinez, J. A., Morched, A. S., Mork, B. A., Parniani, M., Sharshar, A., Shirmohammadi, D., Walling, R. A. and Woodford, D. A., Modeling and analysis guidelines for slow transients - Part III: The study of ferroresonance. IEEE Transactions on Power Delivery, 15, 255-265 (2000).

[2] Carreras, B. A., Lynch, V. E., Dobson, I. and Newman, D. E., Critical points and transitions in an electric power transmission model for cascading failure blackouts. Chaos, 12, 985-994 (2002).

[3] Harb, A. M. and Widyan, M. S., Chaos and bifurcation control of SSR in the IEEE second benchmark model. Chaos Solitons \& Fractals, 21, 537-552 (2004).

[4] Ohta, H. and Ueda, Y., Blue sky bifurcations caused by unstable limit cycle leading to voltage collapse in an electric power system, Chaos Solitons \& Fractals, 14, 1227-1237 (2002).

[5] Andrievskii, B. R. and Fradkov, A. L., Control of chaos: Methods and applications. I. Methods, Automation and Remote Control, 64, 673-713 (2003).

[6] Lu, Z., Shieh, L.S., Chen, G. R. and Coleman, N.P., Adaptive feedback linearization control of chaotic systems via recurrent high-order neural networks. Information Sciences, 176, 2337-2354 (2006).

[7] Liu, D., Ren, H. P. and Kong, Z. Q., Control of chaos solely based on RBF neural network without an analytical model. Acta Physica Sinica, 52, 531-535 (2003).

[8] Heydeman, J., Tripathy, S. C., Paap, G. C. and Sluis, V. D. L., Digital and experimental study of voltage collapse and instability in power system. International Journal of Electrical Power \& Energy System, 22, 303-311 (2000).

[9] Saad, M. S., Hassouneh, M. A., Abed, E. H. and Edris, A. A., Delaying instability and voltage collapse in power systems using SVCs with washout filter-aided feedback. Proceedings of the 2005 American Control Conference (ACC), Portland, OR, 4375-4362 (2005).

[10] Ramirez, G. V. G., Valdes, L. G. V., Medina, M. A., Justo, M. A. and Zaragoza, C. M. A., Facing chaos in parallel active filters using robust nonlinear control. Revista Tecnica De La Facultad De Ingenieria Universidad Del Zulia, 34, 139-147 (2011).

[11] Chen, X. W., Zhang, W. N. and Zhang, W. D., Chaotic and subharmonic oscillations of a nonlinear power system. IEEE Transactions on Circuits and Systems II-Express Briefs, 52, 811-815 (2005).

[12] Coelho, L. D. and Bernert, D. L. D., PID control design for chaotic synchronization using a tribes optimization approach. Chaos, Solitons and Fractals, 42, 634-640 (2009).
[13] McGookin, E. W., Smith, M. D. J., Li, Y. and Fossen, T. I., Ship steering control system optimisation using genetic algorithms. Control Engineering Practice, 8, 429-443 (2000).

[14] Jiao, L. C. and Wang, L., A novel genetic algorithm based on immunity. IEEE Transactions on Systems Man and Cybernetics Part A - System and Humans, 30, 552-561 (2000).

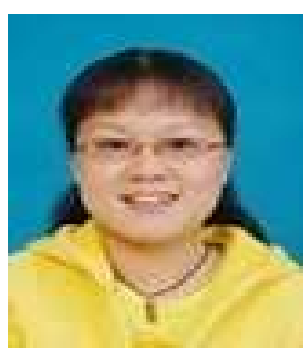

Xiangqun $\mathbf{L i}$, received her Ph.D. degree of electrical engineering technology from China University of Mining and Technology, Xuzhou, China in 2008. She is an associate professor and a master tutor at School of computer science and technology,CUMT. Her current research interests include information processing, data mining and machine learning. She has published 7 books and 5 academic papers.

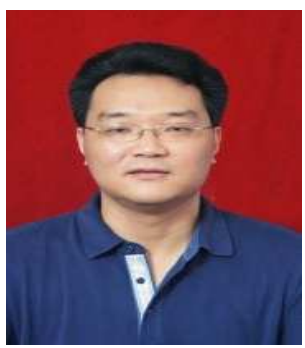

Jianhua Liu received his Ph.D. degree of Power Electronics and Electrical Drive from China University of Mining and Technology, Xuzhou, China in 2008. He is both an associate professor and master tutor at School of Information and Electrical Engineering, CUMT. His current research interests include the smart grid and electrical safety. He has published 12 academic papers.

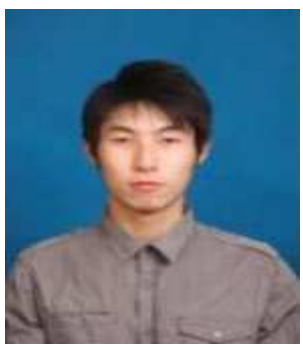

Longyue Yang was enrolled for his Ph.D. degree of electrical engineering technology in China University of Mining and Technology, Xuzhou, China in 2010. He is a Ph.D. candidate at School of Information and Electrical Engineering. His current research interests include the power quality control. He has published 2 academic papers. 